Instituto Internacional de Investigación y Desarrollo Tecnológico Educativo INDTEC, C.A.

DOI: https://doi.org/10.29394/scientific.issn.2542-2987.2017.2.6.8.154-173

OAI-PMH: http://www.indteca.com/ojs/index.php/Revista Scientific/oai

\title{
La Eficacia Publicitaria en las Campañas de Prevención para la Seguridad Vial
}

Autores: Julia Catalina Serrano Cordero Universidad del Azuay, UDA cserrano@uazuay.edu.ec Cuenca, Ecuador José David Arévalo Peña Universidad del Azuay, UDA davitarevalo@gmail.com Cuenca, Ecuador

\section{Resumen}

Este artículo aborda la importancia de los procesos de evaluación de los resultados en las campañas de educación y seguridad vial, éstos, si bien, han alcanzado notables progresos en la praxis, evidencian una falta de información en cuanto a la pertinencia e idoneidad de las herramientas de comunicación en prevención. El objetivo fue validar una campaña de educación y seguridad vial implementada por la Empresa Municipal de Tránsito y Transporte de la ciudad de Cuenca (Ecuador), en el año 2014, para lo cual se usaron técnicas cualitativas y cuantitativas, escogiendo una muestra probabilística estratificada de 304 universitarios, (edad: 15-39). Los instrumentos de recolección de datos fueron: grupo focal, cuestionario y registros estadísticos, se interpretaron mediante el análisis de contenido y la estadística descriptiva. Los hallazgos indican que la percepción más frecuente acerca de las causas de los accidentes es: consumo de alcohol, exceso de velocidad, uso del celular. Asimismo, la campaña "Mejor Prevenir" obtuvo un nivel de reconocimiento generalizado, siendo los más jóvenes quienes recibieron mayor influencia de su contenido comunicacional. Resultados que corroboran que los métodos de motivación y persuasión sí inciden en los cambios de actitud, lo que influye en la transformación hacia una cultura de la prevención vial.

Palabras clave: evaluación; prevención de accidentes; campañas de educación y seguridad vial. 


\title{
Advertising Efficiency in Road Safety Prevention Campaigns
}

\begin{abstract}
This paper addresses the importance of the results evaluation processes in the education and road safety campaigns, although they have achieved remarkable progress in praxis, evidenced a lack of information as to the relevance and suitability of the tools of communication in prevention. The objective was to validate an education and road safety campaign implemented by the Municipal Transit and Transport Company of the city of Cuenca (Ecuador) in 2014, for which qualitative and quantitative techniques were used, choosing a stratified probabilistic sample of 304 university students, (age: 1539). The data collection instruments were: focus group, questionnaire and statistical records, interpreted through content analysis and descriptive statistics. The findings indicate that the most frequent perception about the causes of accidents is: alcohol consumption, speeding, cell phone use. Likewise, the campaign "Best to Prevent" obtained a level of generalized recognition, but it was the younger ones who received more influence of their content of communication. Results that corroborate that the methods of motivation and persuasion do affect the attitude changes, which influences the transformation towards a culture of road prevention.
\end{abstract}

Keywords: evaluation; accident prevention; education and road safety campaigns.

Date Received: 29-04-2017

Date Acceptance: 03-06-2017 


\section{Introducción}

La inobservancia de las leyes fundamentales de tránsito para la prevención de accidentes es un problema grave a nivel local, nacional, regional y mundial. Según estudios realizados por organismos internacionales como la Organización Mundial de la Salud (OMS, 2004), el número de fallecimientos causados por accidentes de tránsito es de 1.2 millones cada año. En América Latina la severidad del problema es mayor aún debido al apresurado incremento del tamaño del parque automotriz en los últimos años.

Como consecuencia del aumento de vehículos se evidencia la necesidad de implementar esfuerzos dirigidos a impulsar la educación vial de los conductores en general y de los conductores jóvenes de manera particular puesto que, tanto su falta de experiencia para conducir vehículos, como su estilo de vida, les convierte en el objetivo idóneo para ser susceptibles de este tipo de campañas, las estimaciones y proyecciones indican que "en los países de América Latina y el Caribe, entre el año 2000 y el 2020, el número de víctimas mortales por el tránsito ascenderá en un 48\%" (Planzer, 2005: 6).

Frente a esta problemática, entidades públicas tanto como privadas, con la asistencia de agencias de publicidad, implementan con regularidad campañas de prevención y educación vial basadas más en la experiencia, producto de un proceso de aprendizaje de aciertos y errores, que en procesos sistemáticos de evaluación y validación de resultados que apoyen y respalden la toma de decisiones, considerando además que este tipo de campañas no buscan vender un bien, sino persiguen generar cambios en las actitudes de las personas. En consecuencia, la mayoría de las campañas de seguridad vial, ejecutadas en función de esta problemática, son realizadas con poca información, y son escasas las evaluaciones que permiten determinar su eficacia una vez concluida la fase de implementación.

En el caso particular del Ecuador y, luego de analizar la información proporcionada por la Agencia Nacional de Tránsito (ANT, 2015), según la cual 
Pichincha es la provincia con mayor número de accidentes, habiéndose registrado un total de 8.604 siniestros en el año 2014, seguida de Guayas con 6.372 siniestros y Azuay (provincia donde se lleva a cabo esta investigación) en tercer lugar con 1.104 (ANT, 2015), se evidencia la necesidad de generar acciones que aporten a la reducción de accidentes de tránsito, número de heridos y fallecidos, además de daños materiales.

Así pues, en vista de las altas cifras de accidentes de tránsito, la Empresa Municipal de Tránsito y Transporte de la ciudad de Cuenca (EMOV), lanza en el año 2014 la campaña "Mejor Prevenir", con el objetivo de reducir los accidentes de tránsito, atacando las principales causas: conducir en estado de embriaguez y con exceso de velocidad. Para ello, se opta por estrategias comunicacionales que impulsan la participación, concienciación, socialización y educación ciudadana en materia de normas básicas de tránsito y en causas fundamentales que inciden directamente en la accidentabilidad en la ciudad.

Considerando estos antecedentes resulta pertinente señalar que el objetivo de esta investigación es sistematizar el proceso de evaluación de la eficacia de la campaña de educación vial "Mejor Prevenir", con el propósito de identificar las acciones más eficaces para generar un cambio de comportamiento en los conductores.

\section{Referentes Teóricos}

Este proyecto busca medir las repercusiones de la campaña "Mejor Prevenir", a partir de un sustento académico, con la intención de determinar la eficacia de la misma. Entonces, es relevante dejar claro que para la evaluación de toda campaña se deben tomar en cuenta los objetivos planteados, debido a lo cual es básico considerar la eficacia publicitaria como el grado de consecución de los objetivos marcados para conseguir la finalidad de la campaña. De igual manera, una campaña publicitaria -una vez implementadas todas las acciones planificadas- logra sus objetivos en la medida en que se 
conecte correctamente con su público objetivo y éste responda al mensaje de la manera esperada, por lo que su eficacia se pondera en función de cobertura, frecuencia y percepción. Entonces, si el público objetivo lleva a cabo la conducta que el creativo publicitario ha pretendido, las técnicas persuasivas han logrado su meta (Senovilla, 2014).

De acuerdo con la información proporcionada por MakmaFill Cía. Ltda. (2014), agencia que desarrolló la campaña "Mejor Prevenir", para el cumplimiento de los objetivos se buscó la mitigación de prácticas ciudadanas que atenten contra los derechos de los actores de la movilidad del cantón Cuenca; y la motivación para cumplir la normativa vigente para una ordenada movilidad cantonal, mediante los procesos de comunicación, sensibilización y educación ciudadana en temas de seguridad vial y responsabilidad social. Para lo cual se implantó el slogan "Mejor Prevenir", que a largo plazo apunta a un cambio de las prácticas ciudadanas para la efectiva vigencia del respeto social, así como llegar a todos los actores de la movilidad: peatones, usuarios de la transportación, ciclistas, conductores del transporte público y privado.

Para determinar si las acciones fueron eficaces o no, se analiza la información presentada por la Empresa Municipal de Transporte de Cuenca (EMOV, 2014), con el fin de establecer la eficacia con base en el planteamiento estratégico, los medios utilizados, el público objetivo, el alcance y cada una de las actividades de sensibilización. Sin embargo, para evaluar de la eficacia se tiene que profundizar en la manera de pensar del público objetivo.

Por otra parte, es relevante tener en cuenta el papel activo del receptor en la significación final que atribuye al mensaje, la cual determina sus respuestas. Al respecto es pertinente referirse a Lerma (2014), quien afirma que desde la Psicología Cultural es necesario "asumir la publicidad como dispositivo de subjetivación y reconocer su valor en la construcción y/o modelación de la mente, dejando claro que los medios, las pantallas, y básicamente, la publicidad contenida en ellas, son los principales referentes 
para la construcción de significados y sentido en nuestra cultura" (pág. 14). De ahí que es importante hacer un análisis desde el punto de vista cognitivo y estudiar la atención, el recordatorio, el conocimiento y la comprensión del receptor.

Una campaña publicitaria tiene varios elementos susceptibles de análisis, entre ellos los elementos simbólicos, los elementos gráficos, el copy, la fotografía, el logotipo de la campaña, el logotipo institucional de la empresa ejecutora, por nombrar algunos. Adicional a eso, hay que considerar que no todos los soportes publicitarios son iguales, en cada caso se debe adaptar y jerarquizar la información, para cada problemática se presentan diferentes variables. Por ejemplo, en las acciones que persiguen concienciar a la población para que no conduzcan en estado de embriaguez se debe trabajar con base en estadísticas. Según la EMOV (2014), se entiende que el mayor número de detenidos en el Centro de Infractores de Tránsito son hombres. Por otro lado, al buscar la concienciación en el uso adecuado del celular al conducir, los porcentajes varían pues tanto hombres como mujeres incurren en cometer infracciones al respecto.

Hay que mencionar, además, que se considera para esta evaluación un análisis con un componente emocional. Papí (2011a), plantea que "el bloque afectivo, mide el tipo de actitud que es capaz de generar un estímulo publicitario" (pág. 249). Se abordan seres emocionales que responden de acuerdo con lo que sienten; por ello, al momento de realizar campañas de prevención, es relevante saber cuáles son los factores motivacionales que pueden inducir a un cambio de conducta, así también se debe conocer el estado emocional que lleva al individuo al incumplimiento de las normas.

Generalmente, se considera que lo más conveniente es intensificar las multas y los operativos de control, como si fueran la única manera de controlar las infracciones. Sin embargo, las respuestas que se buscan en este tipo de campañas no es que la gente cambie por miedo a la sanción, sino que tome 
conciencia, que reflexione, que se sensibilice. Al tratarse de una preocupación social es importante apelar a la responsabilidad de las personas para que lleven el mensaje y sean ejemplo, que se motiven a ser agentes de cambio.

Son esas personas sensibilizadas quienes "deben aceptar y adoptar las conductas y las acciones de desarrollo que se llevan a cabo a fin de cumplir con los objetivos planteados" (Solano, 2015: 44). De allí que se busca generar acciones que incidan en el desarrollo de la conciencia de los individuos, para que actúen por convicción propia y no por temor a las sanciones legales 0 imposiciones.

De modo que es necesario estudiar la respuesta del receptor y conocer cuáles son sus acciones con respecto al mensaje, por tanto, se considera "abordar el análisis de forma conativa, basados en la respuesta de los individuos para actuar en la dirección pretendida" (Papí, 2011b, pág. 249). Como resultado, se debe conocer si las personas que fueron receptores de la campaña realmente se sensibilizaron o educaron, a tal punto de realizar acciones positivas que evidencien un cambio de actitudes y conducta.

Wells, Moriaty y Burnett (2007: 97), proponen un modelo compuesto por seis tipos de respuestas que, a diferencia de los autores antes mencionados, abarca la persuasión y la asociación para la publicidad eficaz. Estas respuestas y sus objetivos publicitarios son: "Percibir (percepción), Comprender (Cognición), Sentir (Afectivo/Emoción), Conectar (Asociación), Creer (Persuasión) y Actuar (Comportamiento)". Dicho modelo es considerado en esta investigación para el desarrollo de los grupos focales y el diseño de la encuesta, además se constituye en una herramienta para el análisis de los resultados y para la identificación de tendencias.

\section{Materiales y Métodos}

El desarrollo de este estudio requirió de la combinación de métodos cualitativos y cuantitativos. En la fase cualitativa se utilizó un diseño de 
investigación exploratorio con la ejecución de un grupo focal, "este tiene como objetivo primordial proporcionar conocimientos del problema. Asimismo, representa "un espacio de opinión para captar el sentir, pensar y vivir de los individuos, provocando auto explicaciones para obtener datos cualitativos" (Hamuid y Varela, 2012a: 56). La etapa referida se complementa con la fase conclusiva de corte descriptivo, que incluye la aplicación de encuestas y un análisis comparativo de la información existente en las fuentes oficiales.

En opinión de Sánchez (2015), es pertinente acortar la distancia entre "la dualidad cuantitativo-cualitativo pues hoy se adoptan nuevas formas y progresivamente se posibilita el acercamiento, mediante una tercera vía que contempla a ambas posiciones como compatibles y complementarias" (pág. 13). Lo cual implica que, dadas las perspectivas de abordaje de los objetos de investigación en la actualidad, posturas que se caracterizan por la flexibilidad, pluralidad y apertura, es recomendable lograr la complementariedad de los métodos; en consecuencia, el conocimiento científico debe construirse desde visiones globales e integrales.

En lo que concierne a este estudio, la riqueza que aportó la convergencia de las técnicas y procedimientos característicos de los métodos cualitativos y cuantitativos facilitó evaluar la eficacia de una campaña de educación en seguridad vial, de manera que se logró dar respuesta a las siguientes interrogantes:

¿Cuáles son los medios más eficaces para la comprensión y recordación de las campañas sociales?.

¿Cuáles son las acciones de comunicación y publicidad más eficaces para generar un cambio de conducta?

¿Es posible identificar cambios conductuales en función del análisis comparativo de la información estadística correspondiente a los años 2013 y 2014 ?. 
Con respecto a la escogencia de los informantes se consideró una muestra probabilística estratificada, con un error del 5\%, con base en la definición de grupos de edades a las que está enfocada la campaña. "Subgrupo en el que la población se divide en segmentos y se selecciona una muestra para cada segmento" (Hernández, Fernández y Baptista, 2014a: 236). La misma quedó integrada del siguiente modo:

- De 15 a 19 años, se tomaron 73 individuos

- De 20 a 24 años, se seleccionaron 71 sujetos.

- De 25 a 29 años, se escogieron 64 individuos.

- De 30 a 34 años, se tomaron 52 sujetos.

- De 35 a 39 años, se seleccionaron 46 sujetos.

Lo que sumó un total de 304 sujetos muéstrales.

\subsection{Técnicas e instrumentos de recolección de datos}

Para la recolección de la información se emplearon dos (2) técnicas que permitieron hacer converger aportes relevantes de los métodos cualitativos y cuantitativos. Por una parte, se empleó la técnica de la observación, mediante el instrumento conocido como grupo focal (Técnica cualitativa) que facilitó interpretar las actitudes, comportamientos y opiniones con relación a aspectos claves de la seguridad vial. Por otro lado, se manejó la técnica de la encuesta (de corte cuantitativito), cuyo instrumento fue el cuestionario, éste facilitó obtener datos mediante respuestas personales que reflejaron el sentir del encuestado acerca de aspectos fundamentales que determinan las características de la educación vial en la comunidad objeto de estudio.

Asimismo, se requirieron los registros de información de la Empresa Municipal de Tránsito y Transporte de la ciudad de Cuenca (EMOV, 2015), para obtener cifras acerca del total de heridos y fallecidos, así como de los accidentes de tránsito ocurridos. 
En lo que respecta a la técnica de análisis de la información, los datos provenientes del grupo focal se interpretaron mediante el análisis de contenido (procedimiento interpretativo, apoyado en la combinación de categorías), mientras que para comprender los datos obtenidos del cuestionario se usaron técnicas de la estadística descriptiva. En el mismo sentido, los insumos proporcionados por EMOV, se analizaron usando herramientas de la estadística descriptiva.

\section{Análisis de datos y discusión}

\subsection{Técnicas cualitativas: Grupo focal}

Es una técnica que centra su atención en la pluralidad de respuestas obtenidas de un grupo de personas, y es definida como un procedimiento de la investigación cualitativa cuyo objetivo es la obtención de datos por medio de la percepción, los sentimientos, las actitudes y las opiniones de grupos de personas (Hernández, Fernández y Baptista, 2014b, pág. 36).

Esta herramienta de comunicación permite recopilar la percepción de las personas haciendo énfasis en los significados y en las emociones. Como técnica de investigación propicia la obtención de abundante información acerca de un tema previamente definido y en ambientes naturales (Hamuid y Varela, 2012b).

Los participantes del grupo focal son hombres y mujeres universitarios conductores de vehículos, de edades entre los 18 y 35 años. Con ellos se evalúan generalidades de las campañas de educación vial y prevención, y de manera particular, el mensaje publicitario de la campaña 'Mejor Prevenir', entonces, se busca identificar sus percepciones y conocimientos acerca de prevención, normas básicas de tránsito y causas fundamentales que inciden en la accidentabilidad.

Cabe recalcar que la naturaleza cualitativa de la herramienta no permite inferir ni generalizar, siendo su propósito principal obtener puntos de vista. No 
obstante, los hallazgos fueron un apoyo esencial para el desarrollo de la investigación cuantitativa, entre los más relevantes están los siguientes:

- La información es clave para generar una cultura de cambio, pues el conocimiento y la comprensión de normas y reglas de tránsito son indispensables para prevenir accidentes.

- Los operativos de control y sanciones resultan útiles en la medida en que uno y otro son preponderantes para generar cambios a través de la experiencia.

- Las diferentes campañas de educación vial han generado en ellos -en mayor o menor grado- conciencia para prevenir accidentes.

- Con respecto a la campaña 'Mejor Prevenir', se concluye que el mensaje tiene pregnancia pues crea una fuerte conexión con evitar el accidente, se refiere principalmente a causa-efecto y a la comprensión de las consecuencias de los comportamientos no deseados.

- La fuente de información más efectiva son las redes sociales, específicamente Facebook, complementadas con vallas publicitarias.

- Finalmente, con respecto a la percepción de las causas de accidentes más frecuentes, se deduce que distractores como el uso del celular, el exceso de velocidad y conducir en estado de embriaguez son, en este orden, los causales más importantes.

\subsection{Técnicas cuantitativas: Encuestas por muestreo}

Es un cuestionario estructurado de captación que se organiza en preguntas o enunciados que buscan obtener datos específicos acerca de las variables en observación, puede recopilar datos acerca de actitudes, conductas, intenciones formas de vida, datos demográficos, entre otros. (Instituto Nacional de Estadística y Geografía, 2013). 
La decisión de optar por un diseño descriptivo se justifica en la medida en que éste permite describir las percepciones que tiene el público objetivo con la campaña publicitaria y, ayuda a determinar el mensaje percibido de la campaña, así como facilita evaluar los medios utilizados.

El análisis de la opinión de los 304 participantes en esta fase de la investigación permite concluir que:

- El alcohol y el exceso de velocidad son las principales causas de accidentes de tránsito.

- El uso del celular es el distractor más frecuente.

- Las campañas de educación vial en las calles sí motivan a los conductores a auto educarse acerca de las normas y reglamentos vigentes, y a leer los manuales que ayudan a prevenir accidentes.

- El temor al castigo (multa) sí ayuda a prevenir accidentes.

- Las campañas difundidas en medios masivos se confunden con las campañas comerciales, perdiendo impacto y efectividad.

- Los controles con agentes de tránsito son una medida eficaz para prevenir accidentes. Más aún, cuando se los implementa de manera simultánea y complementaria con las campañas de prevención, el efecto de éstas se potencializa debido al temor a la sanción.

- Con respecto a la campaña "Mejor Prevenir", el nivel de reconocimiento de la misma es generalizado; sin embargo, al evaluar la recordación y la eficacia de los mensajes de las piezas gráficas, éstos son considerablemente más altos en los grupos más jóvenes, situación que se explica por la decisión de utilizar redes sociales como el principal canal de difusión.

- Por otro lado, la decodificación del mensaje "Mejor Prevenir" se realiza conforme a los objetivos de la campaña puesto que la mayoría de los participantes en el estudio relacionan la frase con un llamado de 
atención acerca de las consecuencias negativas de conducir en estado de embriaguez, manejar a velocidad excesiva, entre otros.

- Finalmente, es posible sostener que -a criterio- de los encuestados, la campaña "Mejor Prevenir" sí contribuye a disminuir los accidentes de tránsito, aseveración que se corrobora en el análisis comparativo realizado a continuación.

\subsection{Análisis comparativo}

Con la finalidad de enriquecer y validar el estudio se realiza un análisis comparativo con base en la información de accidentes de tránsito, número de heridos y fallecidos de los últimos dos años, para lo cual se toma la información proporcionada por la Empresa Municipal de Tránsito y Transporte de la ciudad de Cuenca (EMOV).

Gráfico № 1. Número de accidentes.

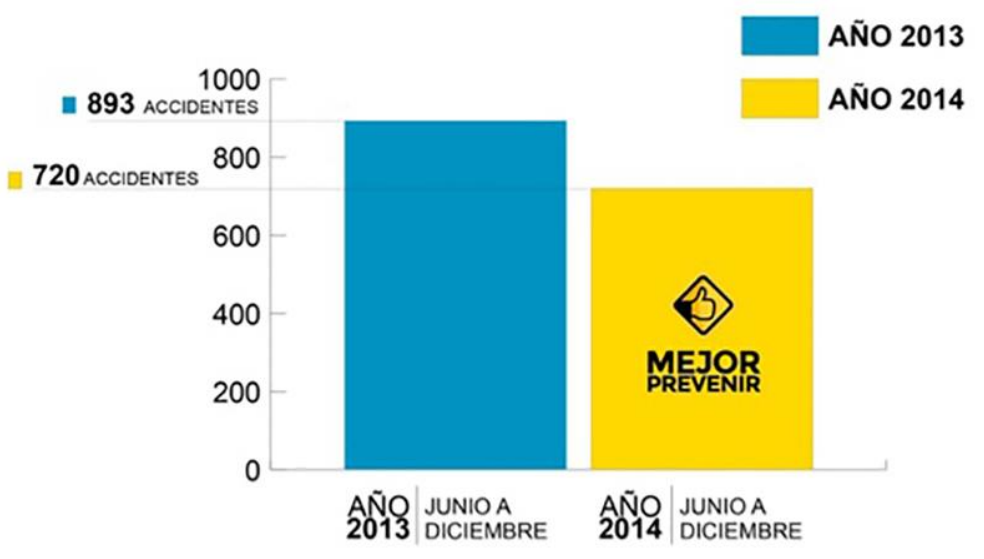

Fuente: EMOV (2015).

Los datos anteriores permiten puntualizar que la campaña "Mejor Prevenir", en su primera etapa (junio-diciembre de 2014), tuvo una repercusión positiva en los destinatarios ya que se observa que para el año 2013, período 
en el que se presentaron 893 accidentes de tránsito, mientras que el año 2014 reporta una cantidad de 720 accidentes, entonces, al comparar estas cifras, se puede constatar que hubo una disminución del 19\% de accidentabilidad. Ello es indicio de que los mensajes publicitarios que alertan acerca de la importancia de cumplir con las normas del tránsito, cumplieron su función persuasiva convenciendo acerca de la necesidad de utilizar la prevención como principal mecanismo que incide en la seguridad vial.

Gráfico № 2. Número de heridos.

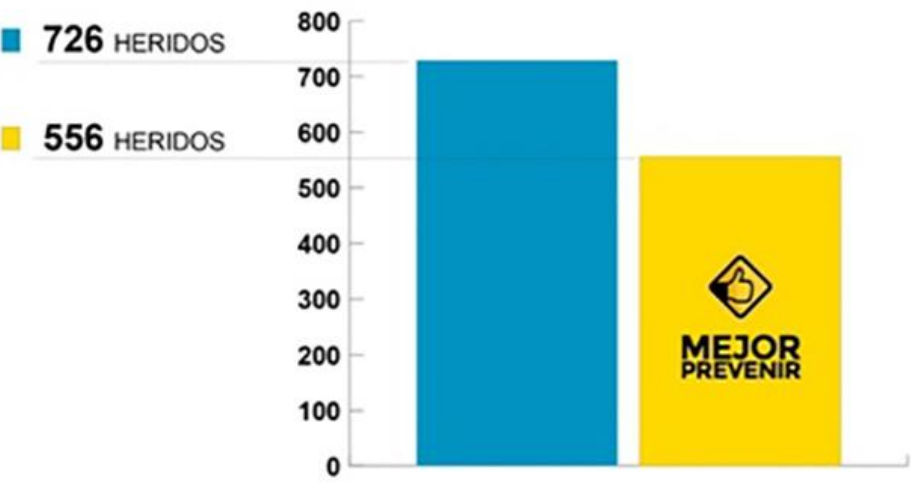

AÑo 2013

AÑo 2014

Fuente: EMOV (2015).

Como se puede comprobar en el gráfico $\mathrm{N}^{\circ}$ 2, el año 2013 arroja 726 heridos, mientras que para el 2014 se computan 556, ello permite inferir que el número de heridos se redujo en un $23 \%$. Datos que hacen posible corroborar la eficacia de la campaña de prevención desarrollada y el logro de los objetivos trazados. Son efectos positivos que sin duda tienen una importante repercusión en diversos ámbitos sociales; por una parte, se disminuyen las consecuencias afectivas y psicológicas que para la familia representa una persona con lesiones físicas en su núcleo y, por la otra, representa una merma en la carga impositiva que para el estado significa la accidentalidad vial, 
dificultad que en muchos países llega a convertirse en un problema de salud pública.

Gráfico № 3. Número de fallecidos.

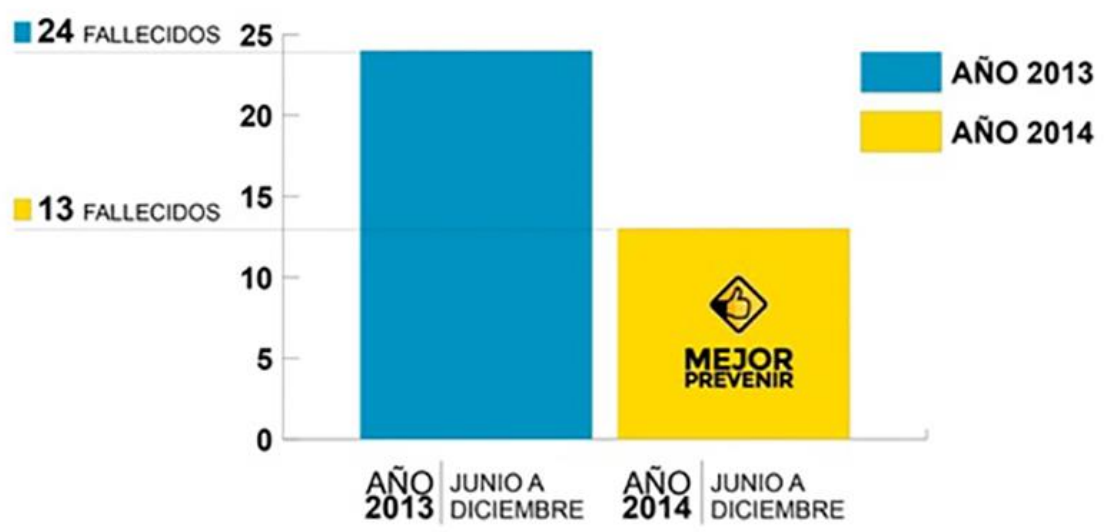

Fuente: EMOV (2015).

En cuanto al número de fallecidos que representa el objetivo principal de toda campaña de seguridad vial, se obtuvo una reducción del $46 \%$, con un número de 24 fallecidos en 2013 y 13 fallecidos en 2014. Información muy significativa en virtud de la trascendencia que tiene preservar la integridad física de los ciudadanos, lo que se concreta en menores impactos sociales y en calidad de vida para la comunidad. En el mismo sentido, es importante dar relevancia al hecho de que uno de los efectos más valorados de las campañas de prevención es lograr que la mortalidad sea controlable por lo cual justifica cualquier esfuerzo al respecto.

Por último, estas cifras permiten confirmar la eficacia de la campaña 'Mejor Prevenir' en la medida en que, conforme se indica en los Gráficos 1, 2 y 3 , al comparar el número de accidentes de tránsito, heridos y fallecimientos, registrados en períodos de tiempo semejantes (junio - diciembre), en el año 2013, versus los registros del año 2014, se evidencia el cumplimento de los 
objetivos planteados en la campaña. Ello debido a que la cantidad de accidentes de tránsito registrados, el número de heridos, así como el total de fallecimientos se reducen en $19 \%, 23 \%$ y $46 \%$, respectivamente.

\section{Conclusiones}

Los hallazgos obtenidos permiten precisar lo siguiente:

Es necesario recalcar la trascendencia que tiene la información en la generación de una cultura de cambio, ya que entender el significado y aprender a respetar las reglas y normas de tránsito contribuye sustancialmente con la prevención de los accidentes. Hay que mencionar además que la manera en que se presentan los contenidos para la comprensión del mensaje está determinada por el tipo de recursos (imágenes, vallas publicitarias, Facebook) empleados, por lo que se deben utilizar los que resulten más eficientes en la captación de la atención del usuario.

También es pertinente establecer que de acuerdo con los individuos para tomar decisiones realizan evaluaciones nuevas o preexistentes del contenido acumulado en los saberes previos. Es así que la retención del mensaje es aún más importante para el cambio de conducta, en virtud de lo cual las tareas de sensibilización, concienciación y educación vial deben ser continuas.

En lo que se refiere a la campaña "Mejor Prevenir", se debe señalar que se ejecutó mediante estrategias comunicacionales de persuasión, las cuales fueron decisivas para motivar la participación, concienciación, socialización y educación ciudadana en materia de normas básicas de tránsito y, sobre todo, para entender las causas fundamentales que inciden directamente en la accidentabilidad del cantón, como lo son: el estado de embriaguez y el exceso de velocidad.

Asimismo, los datos discutidos permiten afirmar que la campaña en referencia alcanzó sus objetivos pues hizo posible reducir accidentes de 
tránsito, por consiguiente, número de heridos y número de fallecidos. De manera que las campañas de educación vial son un recurso determinante para formar a los ciudadanos en el conocimiento de los reglamentos y normas que regulan el tránsito, en consecuencia, crean conciencia acerca de la necesidad de prevenir accidentes.

Finalmente, es valioso resaltar que los hallazgos obtenidos facilitan afirmar que las sanciones tienen efecto en ciertos grupos, pero la sensibilización y la educación (campañas publicitarias) abordada desde un tono proactivo es fundamental para un cambio de cultura y para la transformación de prácticas ciudadanas desde la convicción del individuo. De manera que más allá del conocimiento y de la información, la automotivación es lo más relevante pues si la persona trabaja en su propio comportamiento para cambiar los malos hábitos se puede alcanzar un cambio de conducta positivo que mejore el desempeño ciudadano.

\section{Referencias}

Agencia Nacional de Tránsito (2015). Estadísticas de transporte terrestre y seguridad vial. Recuperado de:

http://www.ant.gob.ec/index.php/noticias/estadisticas\#.Vk9KEt8rJp9

Agencia Makma Fill. (2014). Studio Creativo Makmafill Cía, Ltda. Cuenca, Ecuador.

Empresa Pública Municipal de Movilidad, Tránsito y Transporte de Cuenca (2015). Estadísticas de accidentes de accidentes, heridos y fallecidos por accidentes de tránsito. (2013-2014).

Hamuid, A. y Varela, M. (2012a,b). La técnica de grupos focales. Investigación en Educación Médica. 2(1): 55-60.

Hernández, S., Fernández, C. y Baptista, P. (2014a,b). Metodología de la Investigación. México: McGraw-Hill. 
Instituto Nacional de Estadística y Geografía. (2013). Diseño de Cuestionarios. México: Instituto Nacional de Estadística y Geografía.

Lerma, C. (2014). Desde la publicidad como objeto poco digno de interés académico, a la publicidad como elemento constitutivo de la cultura. Diálogos. De la Comunicación. Julio-diciembre, pp. 1-16.

Organización Mundial de la Salud. (2004). Informe mundial sobre prevención de los traumatismos causados por el tránsito. Recuperado de:

http://www.who.int/violence injury prevention/publications/road traffic/ world report/summary es.pdf

Planzer, R. (2005). CEPAL (Naciones Unidas) Serie: Recursos Naturales e infraestructura. Santiago de Chile: CEPAL.

Papí, N. (2011a,b). La eficacia publicitaria de las campañas sobre la Igualdad de género: análisis de la codificación y del plan de medios. Alicante: Universidad de Alicante.

Sánchez, M. (2013). La dicotomía cualitativo-cuantitativo: posibilidades de integración y diseños mixtos. Campo Abierto, vol. monográfico, pp. 11-30.

Senovilla, S. (2014). Análisis de la comunicación publicitaria de Apple: La construcción de imagen de marca a través de la creatividad. Tesis de grado de Licenciatura no publicada. Universidad de Valladolid: España.

Solano, D. (2015). Estrategias de comunicación y educación para el desarrollo sostenible. Santiago de Chile: UNESCO.

Wells, W., Morirarty S., Burnett, J. (2007). Publicidad: principios y práctica. México DF: Pearson Educación. 


\section{Julia Catalina Serrano Cordero \\ e-mail: cserrano@uazuay.edu.ec}

Nacida en Ecuador. Doctorando en Estudios Latinoamericanos por la Universidad Andina Simón Bolívar, sede en Quito. Máster en Comunicación, mención en Publicidad (California State University), Máster en Dirección de Marketing y Gestión Comercial (Universidad de Barcelona / OBS), Diplomado en Análisis de Datos Complejos (Universidad del Azuay). Profesora Principal de pregrado y posgrado y Directora del Departamento de Posgrados de la Universidad del Azuay. Miembro del Centro de investigaciones sobre el estado, la calidad de la burocracia y política pública. Participante invitado en el seminario "Welfare States, Labour Rights and Basic Income in Latin America" Comparative Research Programme on Poverty (CROP), Brasil (2016). 


\section{José David Arévalo Peña \\ e-mail: davitarevalo@gmail.com}

